\title{
FACE RECOGNITION USING RELATIONSHIP LEARNING BASED SUPER RESOLUTION ALGORITHM
}

\author{
${ }^{1}$ SenthilSingh and ${ }^{2}$ Manikandan \\ ${ }^{1}$ Research Scholar, Anna University, Chennai, India \\ ${ }^{2}$ Department of Electronics, Anna University, Chennai, India \\ Received 2013-01-28; Revised 2013-08-03; Accepted 2014-01-16
}

\begin{abstract}
The face recognition is an application which is for identifying a person from a digital image. The common problem that often occurs while identifying the face from image is due to the low resolution in images especially when it is captured from a long distance. In automated face recognition system, this has always been a challeging problem. To overcome this problem, an approach is proposed to learn the high resolution face and the VLR image face for face. In this new approach the face recognition applications under the VLR problem is designed for good visuality. To create the Very Low Resolution (VLR) image corresponding to each of these High Resolution (HR) images, the HR images are resized to $64 \times 48$ pixels. The Very Low Resolution (VLR) of the face image is lower than $16 \times 12$ pixels. The proposed system is implemented in MATLAB. The performance of the proposed system is tested. The proposed system is highly accurate and extremely fast in processing the image data. Experimental results show that proposed method outperforms existing methods. The new data constraint that measures the error in the HR image space was developed and RLSR was proposed.
\end{abstract}

Keywords:Very Low Resolution (VLR), Super Resolution (SR), Relationship-learning based SR (RLSR), High Resolution (HR), Low Resolution (LR)

\section{INTRODUCTION}

The face recognition system is an application which is used for identifying or verifying a person from a digital image. One way is by comparing the selected face features from the image and a facial database. Several techniques have been implemented to carry out this application. Baker and Kanade developed an approach based on a Gaussian pyramid and Laplacian pyramid model and employed the Bayesian theory to infer the super-resolved face image from the LR and used a steerable pyramid to extract multi-orientation and multiscale information of low-level features from both the input LR face images and HR face images in database.

The increasing number of applications ie personal authentication (e.g., access control, surveillance of people in public places, security of transactions and human-computer interaction) and the availability of low-cost recording devices. Some reliable biometric recognition techniques are fingerprint and iris recognition. By using this technique it is intrusive and the success depends highly on user cooperation. But face recognition is non-intrusive, it is based on images which is recorded by camera. The user is not aware the existence of the face recognition system. The human face is the common characteristic used by humans to recognize other people and personal identification based on facial images is among all biometrics.

In this applications camera is installed in a way but the viewing area is maximized but the face region is very small. To recognize a camera, it is to handle low resolution face image with variations such as pose, illumination and occlusion.

Super-resolution from a single image, by Glasner et al. (2009) proposed a unified framework for combining the two general approaches of super resolution. It show how this combined approach can be applied to obtain super resolution from as little as a single image (with no database or prior examples).

Jia and Gong (2008) proposed the idea to learn a map from input low resolution images to target high resolution images based on example pairs of input and output images.

Corresponding Author: SenthilSingh, Research Scholar, Anna University, Chennai, India 
Deriving a High-Resolution (HR) image from the Low-Resolution (LR) one or a sequence of LR images provides a solution to these applications, which is known as the Super Resolution (SR) imaging technique Cristbal et al. (2008). The SR algorithms can be categorized into two classes, i.e., multi-frame based approach Qin et al. (2009) and single-frame based approach, which is also called learning-based approach. In the multi-frame based approach, the HR image is derived from several LR observations of the scene, which are typically aligned with sub-pixel accuracy; while in the learning-based approach an image database, which includes LR and HR image pairs, is used to infer the HR image from its corresponding LR input. Existing Algorithm is proposed for Super Resolution (SR) for face image. By applying the algorithm the low resolution face image is reconstructed into high resolution face image. But this proposed algorithm works well when the image is in good illumination.

Zou and Yuen (2012) proposed the VLR problem using RLSR Algorithm for $16 \times 16$ pixels. The face super resolution algorithm to address recognition of low resolution face image with nonlinear variations. The proposed method learns the nonlinear relationship between low resolution face image and high resolution face image in (nonlinear) kernel feature space Zou and Yuen (2010).

When a person is not close to the camera then the resolution for the face image is less than $16 \times 12$ pixels. But it gives limited information and much information is lost.

To solve the VLR problem is to recover the missed information of the face image. Existing algorithms are classified into two approaches namely maximum a posteriori and example-based approaches. These two approaches perform error evaluation for the reconstructed. HR images, which is called a data constraint. The data constraint is used for comparing the images by calculating the distance in the low-resolution image space for Super Resolution Processing.

This study proposed a technique, RelationshipLearning based SR (RLSR) (SenthilSingh and Manikandan, 2012) approach for solving the VLR problem and implemented by using MATLAB. The proposed scheme addresses the Very Low Resolution problem in face recognition where the resolution of the face images to be recognized is lower than $16 \times 12$ pixels. This proposed scheme explains the novel approach to learn the relationship between the high-resolution image space and the VLR image space for face SR.

\subsection{Steps in Face Recognition}

Figure 1 discuss the generic face recognition system consists of three main processing steps:

- Face detection means detecting and separation of face region from the given face image or video
- Feature extraction means which identifies and extract features of the submitted images. Features are local features such as lines, points, or facial features such as eyes, nose and mouth

- Face Recognition is by matching input image against the faces in database

\subsection{Super Resolution}

Super Resolution (SR) is a technique that enhances the resolution of an imaging system. Super-resolution is a growing technique as a purely computational means to increase imaging sensors performance. It is unrealistic to assume that the super resolved image can recover the original scene $\mathrm{o}(\mathrm{x}, \mathrm{y})$. A reasonable goal of $\mathrm{SR}$ is a discrete version of $\mathrm{o}(\mathrm{x}, \mathrm{y})$, which has higher spatial resolution than the resolution of the LR images and which is free of the volatile blurs (deconvolved). The standard SR approach consists of sub pixel registration, overlaying the LR images on an HR grid and interpolating the missing values.

Though many techniques for image enhancement have been proposed, many realistic problems have not been solved satisfactorily. When an image is magnified or upsampled many times, blur or mosaic phenomena tend to occur thus diminishing the quality of the image. In case of wireless/remote surveillance, the resolution of obtained video is quite low due to limited bandwidth requirement in transmission or large data real time transmission and thus details for the people or object are not clear. In order to solve these problems, the Super Resolution techniques are employed. The idea is to construct a HR from a single or multiple LR images. In terms of pixels, the size of HR image is larger than LR image and the enhance techniques can magnify the LR image and increase image details, making the magnified image close to the HR origin image. These techniques have become a popular research topic due to its broad applications in face recognition and surveillance.

The face similarity in the Very Low Resolution (VLR) image space cannot be an actual face similarity of HR face images. The existing SR algorithms may not be employed under the VLR problem. To solve the VLR problem, a Relationship Learning-based SR (RLSR) approach is proposed in this study. This algorithm makes use of the information available during the training phase and proposes a new learning-based face SR approach. Unlike most of the existing methods recovering the images directly from the VLR images and the training images, this algorithm first determines relationship between VLR and HR image spaces and then applies it on VLR images to recover the HR ones. 


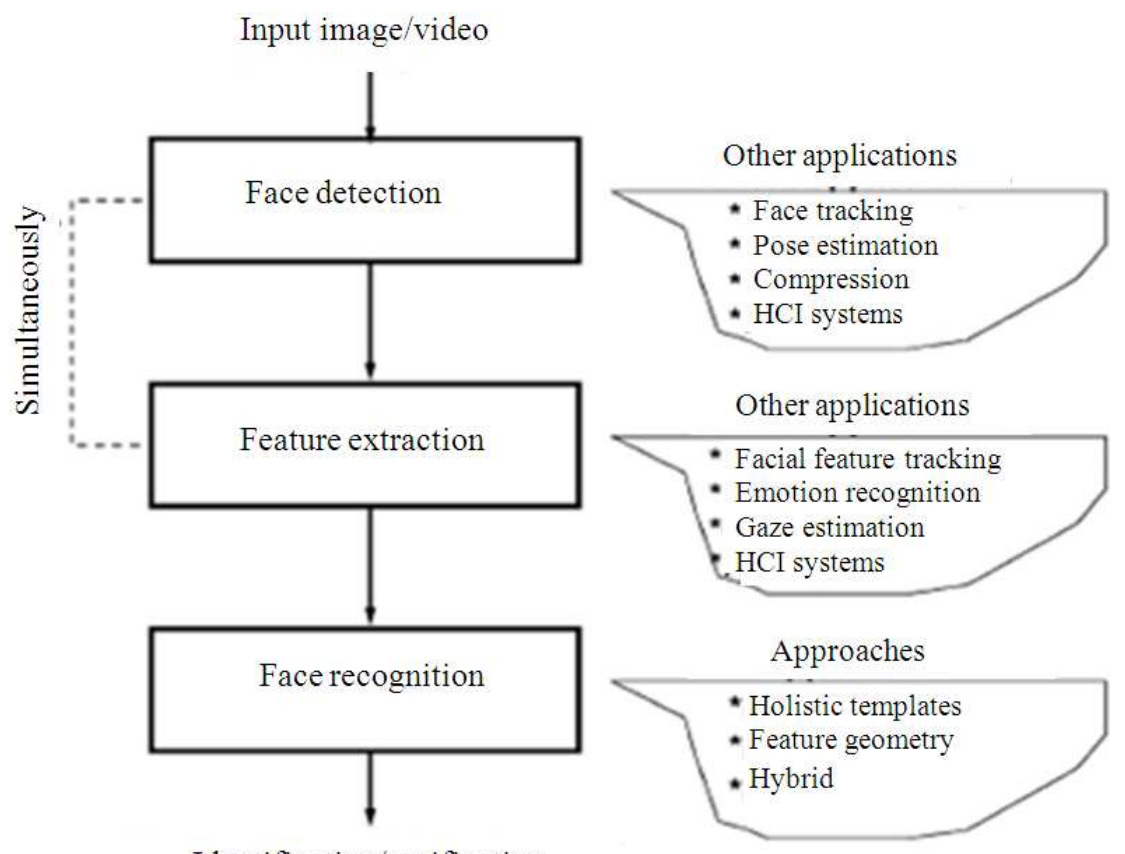

Identification/verification

Fig. 1. Configuration of a generic face recognition system

The proposed new approach offers three additional advantages. (i) SR algorithm can recover images with more details and handle the VLR problem better. (ii) The linearity clustering ensures data linearity in each cluster. Therefore, the linearity clustering-based relationship learning method can handle complex nonlinear case. (iii) The determined relationship, $\mathrm{R}$ is generic for all VLR testing face images.

\subsection{Proposed System}

The proposed SR algorithm can recover images with more details and handle the VLR problem better. The linearity clustering ensures data linearity in each cluster. Therefore, the linearity clustering-based relationship learning method can handle complex nonlinear case.

\subsection{RLSR Algorithm}

There are several algorithms that exist to convert a Low Resolution image into a High Resolution image. But these methods fail when a Very Low resolution image is used. Hence an RLSR algorithm is proposed. The Fig. 2 gives the overall block diagram of the algorithm.

\subsection{Training Phase}

The training phase consists of two steps, namely, linearity clustering and relationship learning. In the first step, a clustering algorithm is proposed as a preprocessing step. After clustering, the Very Low Resolution (VLR)-
High Resolution (HR) image pairs in each cluster are nearly linear, i.e., the relationship can be approximately represented by a matrix. In the second step, the relationship mapping from the VLR to the HR face image spaces within the cluster is determined.

\subsection{The Database}

All the images are taken against a bright homogenous background with the subjects in an upright, frontal position. The files are in JPEG format. The size of each image is $64 \times 48$ pixels, with 256 grey levels per pixel. Both male and female subjects are present in this database. To create the Very Low Resolution (VLR) image corresponding to each of these High Resolution (HR) images, the VLR images are resized to $16 \times 12$ pixels. To create the Very Low Resolution (VLR) image corresponding to each of these High Resolution (HR) images, the HR images are resized to $64 \times 48$ pixels. A test image pair is shown below Fig. 3.

\subsection{Linearity Clustering}

Clustering means assigning a set of objects into groups. The objects in the same cluster are more similar to each other than to those in the other clusters. Depend upon the dataset the appropriate clustering algorithm and parameter settings is done. A linearity clustering algorithm is proposed to ensure that the clustered training image pairs have a linear relationship. 


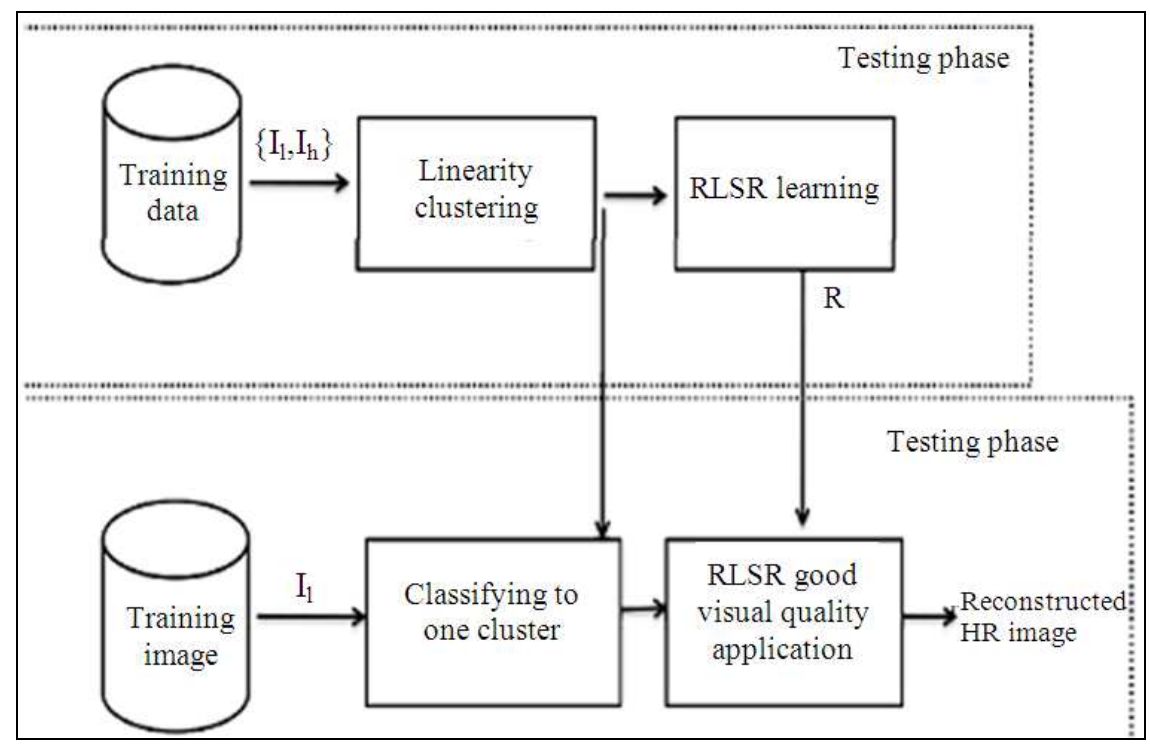

Fig. 2. Block diagram of RLSR

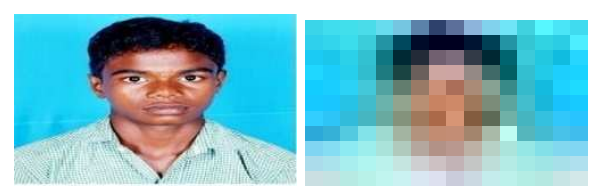

Fig. 3. HR-VLR image pair

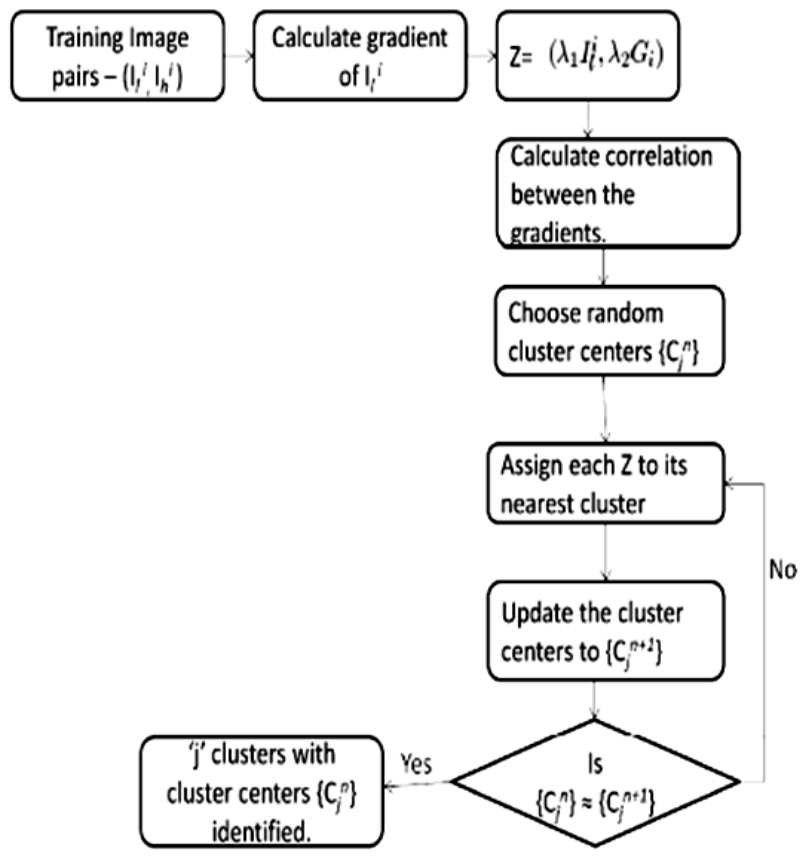

Fig. 4. Block diagram of the clustering algorithm
This clustering algorithm reduces the complexity of the relationship learning process. The Fig. 4 shows the steps involved in the clustering algorithm.

Maximizing the linearity of the clustered data pairs is equivalent to minimizing the difference of the gradient between the data pairs. Thus the clustering algorithm utilizes the following two parameters: (a) The gradient of the low resolution image (b) The low resolution image.

The contribution of the above two parameters is balanced by two constants- $\lambda 1$ and $\lambda 2$.

Figure 5(a) shows the HR images with their corresponding very low resolution image in the database. Once subjected to the linearity clustering algorithm, the images showing maximum similarity are clustered together. This is shown in Fig. 5(b).

\subsection{Relationship Learning}

In RLSR algorithm, cluster holds the training image pairs (VLR-HR). Assume the relationship R be the mapping between VLR to HR with in the cluster, then Ihi = R (Ili). After assuming $\mathrm{R}$ then apply $\mathrm{R}$ to the VLR image to recover the HR image. A matrix $R$ is used after clustering to represent relationship mapping $R$. The relationship operator R can be derived as R = inverse (LR)*HR. From this, the relationship matrix corresponding to each of the image pair is determined. The linear clustering aids in obtaining a unique relationship operator for each of the cluster. So, the relationship operators of the images in a particular cluster are extracted. The relationship operator corresponding each cluster-Rcluster is selected such that reconstruction error is minimum. 


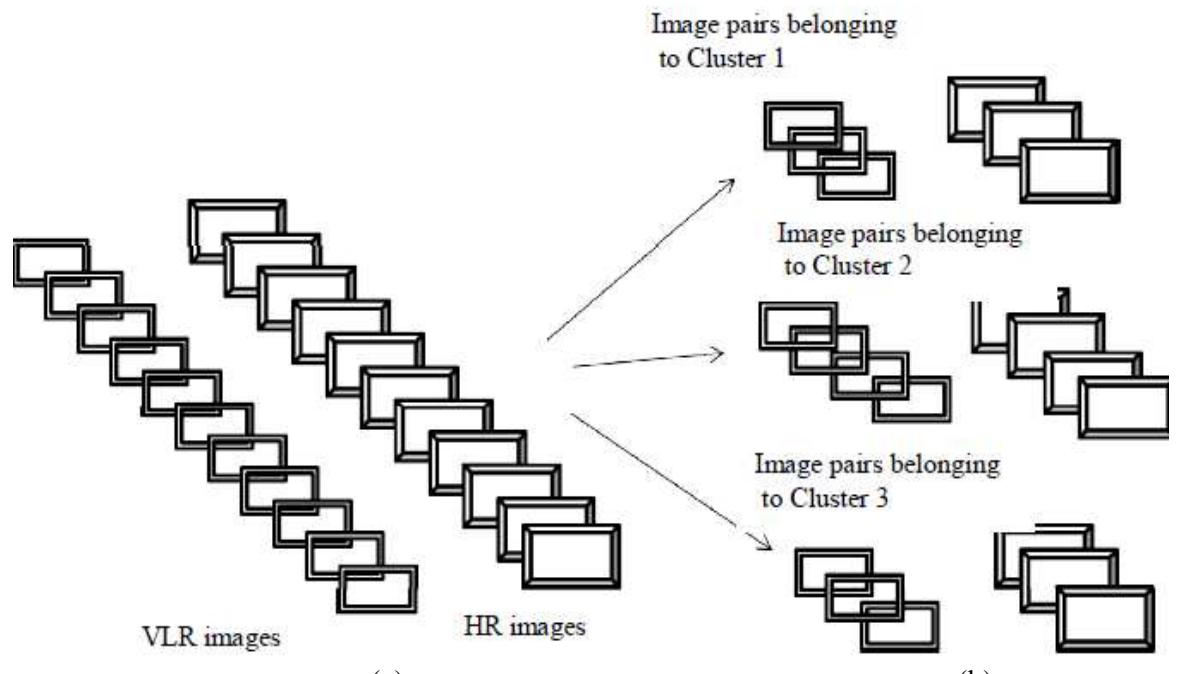

(a)

(b)

Fig. 5. (a) VLR-HR image pairs in the database (b) Clustered image

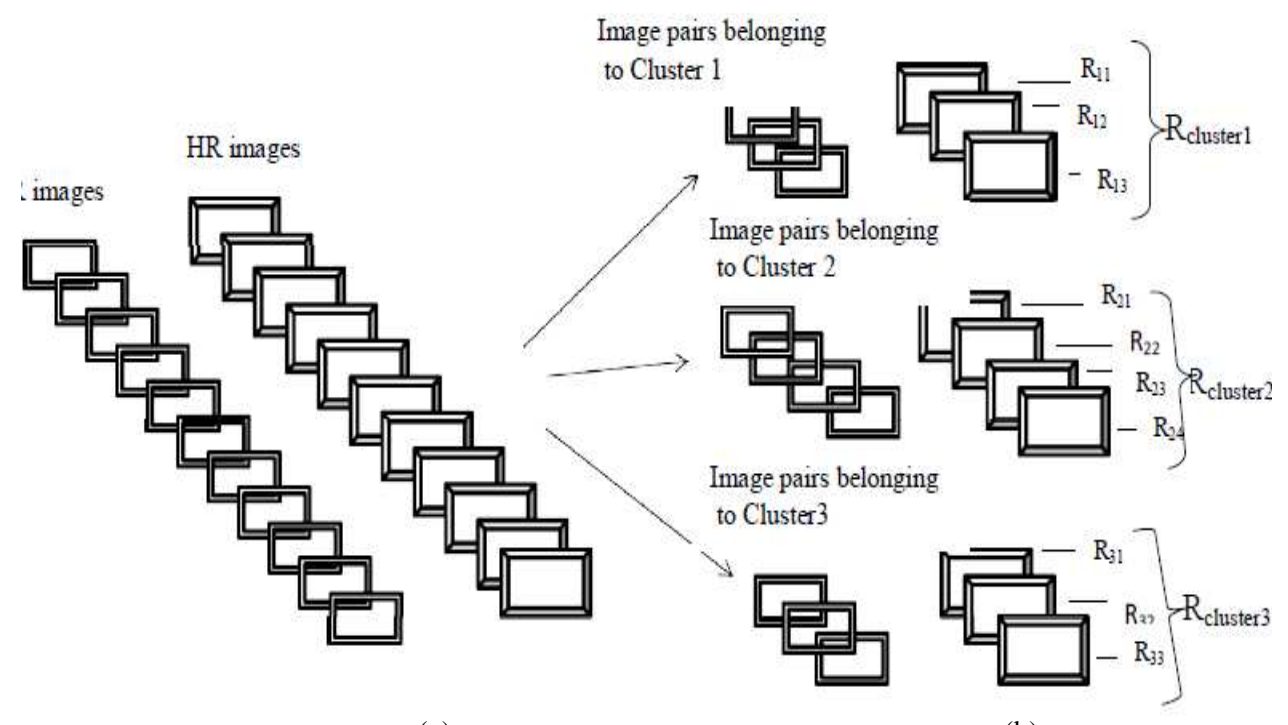

(a)

(b)

Fig. 6. Pictorial representation of the relationship operator- $R_{\text {cluster }}$, corresponding to each cluster. (a) VLR-HR image pairs in database (b) Clustered image pairs used to generate $R_{\text {cluster }}$

Figure 6 gives a pictorial representation of how the unique relationship operator-Rcluster, corresponding to each cluster is obtained. The straight forward method for this is minimising the reconstruction error. The reconstruction error is measured in the HR image space by the data constraint e R:

$$
\mathrm{eR}(\mathrm{Ih})=\left\|\mathrm{Ih}-\mathrm{Ih}^{\prime}\right\|
$$

Here, Ih and Il represent the HR and VLR image respectively. A minimum mean square error is employed to learn $\mathrm{R}$ is represented as:

$$
\operatorname{minR} 1 / \mathrm{N} \Sigma \| \text { Ihi- RIli } \|
$$

Using the above two equations the learning process ensures that it incurs minimum reconstruction error. 


\subsection{Testing Phase}

The dimensions of the test image is $16 \times 12$ pixels with 256 gray levels. For testing VLR image is given as a test image. Gtest is used for calculating he gradient of the image. The parameter of the clustering algorithm used in the training phase is gradient. To classify the VLR image into the appropriate cluster 'i', Gtest is used. After identifying the cluster ' $\mathrm{i}$ ', for input testing image the corresponding R cluster-i is applied.

\section{RESULTS AND DISCUSSION}

The results in MATLAB the training images in the database are grouped into 3 clusters and outputs are displayed. The time complexity for the two relationship learning procedures is also compared.

\subsection{Simulation of Linear Clustering}

The training images on which clustering algorithm was applied is shown below in Fig. 7. The well aligned training images are normalized to the resolutions of $64 \times 48(\mathrm{HR})$ and $16 \times 12(\mathrm{LR})$. Only frontal images are used with different poses.

The number of clusters is chosen to be three and the following Fig. 8a-c show the cluster membership of each image.

The Fig. 9 shows the input query image. The well aligned training images are normalized to the resolutions of $64 \times 48$ (HR) and 16×12 (LR). Only frontal images are used with different poses.

The reconstruction of the HR image from the VLR by the proposed RLSR algorithm. If the number of Clusters $=5$, the images in the training database is clustered as shown below.

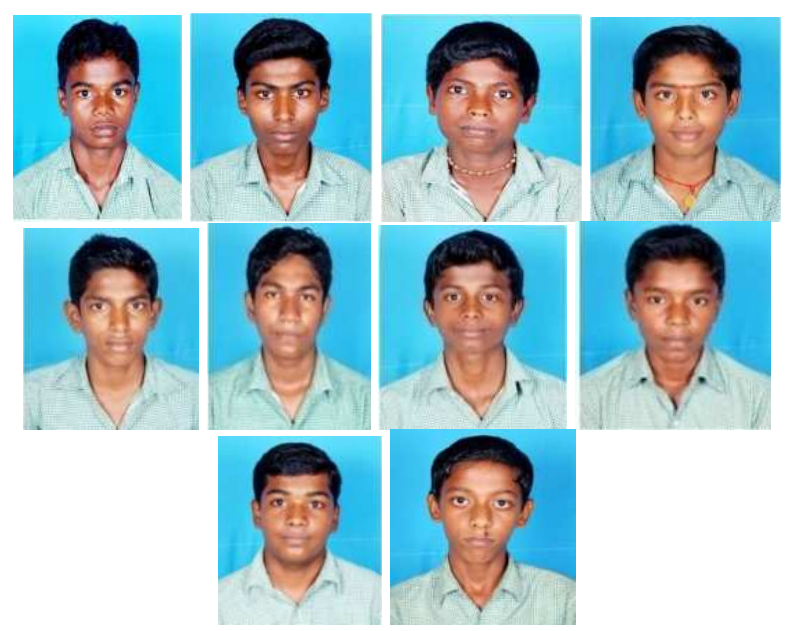

Fig. 7. Sample of training images

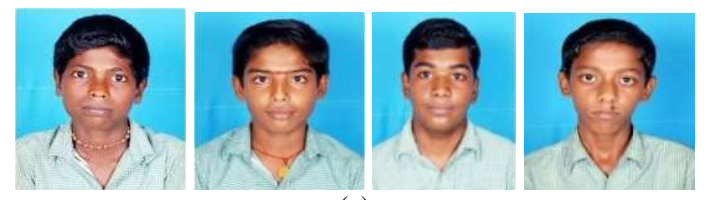

(a)

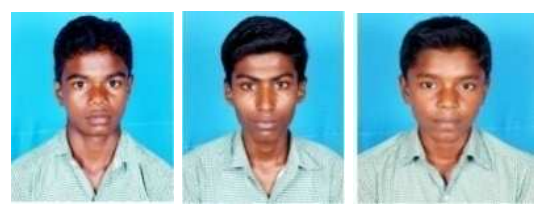

(b)

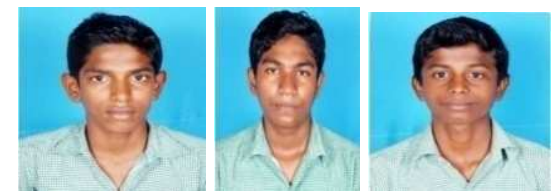

(c)

Fig. 8. (a) Images in Cluster 1 (b) Images in Cluster 2 (c) Images in Cluster 3

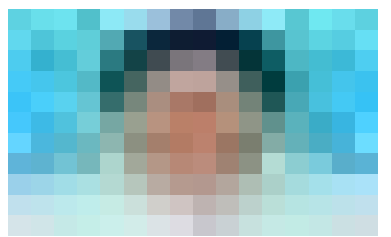

Fig. 9. VLR Test image

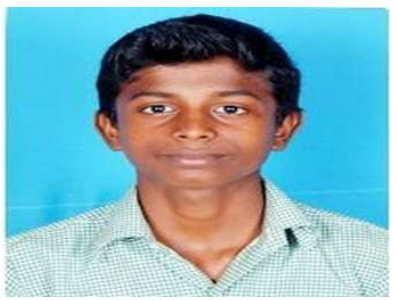

Fig. 10. Reconstructed output HR image

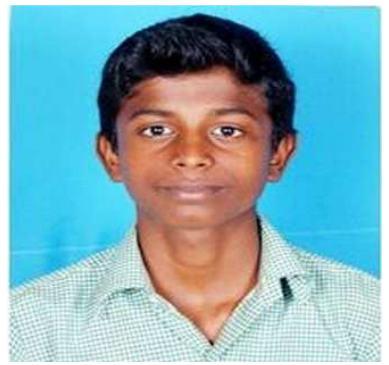

Fig. 11. Reconstructed output HR image 
Table 1. Cluster index table with 5 clusters

\begin{tabular}{ll}
\hline Cluster Index & Image indices \\
\hline Cluster & 11 \\
Cluster & $5,7,8$ \\
Cluster & 6 \\
Cluster & 1,2 \\
Cluster & $3,4,9,10$ \\
\hline
\end{tabular}

Table 2. Cluster index table with 10 clusters

\begin{tabular}{ll}
\hline Cluster index & Image indices \\
\hline Cluster1 & 7 \\
Cluster2 & 1 \\
Cluster3 & 2 \\
Cluster4 & 6 \\
Cluster5 & 9 \\
Cluster6 & 5,8 \\
Cluster7 & 4 \\
Cluster8 & 10 \\
Cluster9 & 11 \\
Cluster10 & 3 \\
\hline
\end{tabular}

Table 3. Comparison of time complexity

\begin{tabular}{lcr}
\hline Procedure & Database size $=11$ & Database size $=21$ \\
\hline Average R & 3.50 & 6.23 \\
Minimum error R & 146.56 & 500.30 \\
\hline
\end{tabular}

The Table 1 in which each row represents a cluster group and their elements indicate the indices of the images present in the database. The reconstruction of the VLR test image, when the training images are grouped into five clusters is shown in Fig. 10.

The PSNR of this reconstructed image is computed to be $23 \mathrm{~dB}$.

If the number of Clusters $=10$, the images in the training database is clustered as shown below.

The Table 2 where each of the rows represents a cluster and its elements represent the indices of the images present in the database. The reconstruction of the VLR test image, when the training images are grouped into ten clusters is shown below in Fig. 11.

The PSNR of this reconstructed image is computed to be $30.8 \mathrm{~dB}$.

The code execution time in MATLAB for the two different Relationship learning procedures adopted in our algorithm is compared below for different database sizes. The values of time mentioned in the Table $\mathbf{3}$ are in seconds.

\section{CONCLUSION}

In this proposed System the face recognition problem in VLR was discussed. To rectify this problem, RLSR algorithm was proposed for good visual quality applications. A new data set was developed for measuring the error in the HR image. In the experimental results it shows that the number of clusters in the algorithm increases, the results obtained are with good visual quality. But, as the number of clusters approaches the number of images in the database it causes overlearning (learning of redundant data). The experimental result cosumes less time in averaging relationships in each cluster than the relationship operator got by minimizing the reconstruction error.

\section{REFERENCES}

Cristbal, G., E. Gil, F. Sroubek, J. Flusser and C. Miravet et al., 2008. Super resolution imaging: A survey of current techniques. Proceedings of the Advanced Signal Processing Algorithms, Architectures, Implementations XVIII, Sep. 3-3, SPIE, San Diego, California, USA., pp 0C1-0C18. DOI: $10.1117 / 12.797302$

Glasner, D., S. Bagon and M. Irani, 2009. Superresolution from a single image. Proceedings in IEEE 12th International Conference in Computer Vision, Sept. 29-Oct. 2, IEEE Xplore Press, Kyoto, pp: 349356. DOI: 10.1109/ICCV.2009.5459271

Jia, K. and S. Gong, 2008. Generalized face superresolution. IEEE Trans. Image Process., 17: 873886. DOI: $10.1109 /$ TIP.2008.922421

Qin, F.Q., X.H. He and W. Wu, 2009. Video superresolution reconstruction based on subpixel registration and iterative back projection. J. Electr. Imag., 18: 1-16. DOI: 10.1117/1.3091936

SenthilSingh, C. and M. Manikandan, 2012. Design and Implementation of an FPGA-based real-time very low resolution face recognition system. Int. J. Adv. Inform. Sci. Technol., 7: 59-65.

Zou, W.W.W. and P.C. Yuen, 2010. Learning the relationship between high and low resolution images in kernel space for face super resolution. Proceedings of International Conference in Pattern Recognition, Aug. 23-26, IEEE Xplore Press, Istanbul, pp: 1152-1155. DOI: 10.1109/ICPR.2010.288

Zou, W.W.W. and P.C. Yuen, 2012. Very low resolution face recognition problem. IEEE Trans. Image Process., 21: 327-40. DOI: 10.1109/TIP.2011.2162423 